\title{
Effect of Inner Rod Tilting on the Performance of a Cylindrical Differential Electrical Mobility Analyzer (DEMC)
}

\author{
Thamir Alsharifi ${ }^{1,2}$, Da-Ren Chen ${ }^{1 *}$ \\ ${ }^{1}$ Particle Laboratory, Department of Mechanical and Nuclear Engineering, Virginia Commonwealth University, \\ Richmond, VA 23284, USA \\ ${ }^{2}$ University of Baghdad, Baghdad, Iraq
}

\begin{abstract}
The objective of this study was to investigate the effect of inner rod tilting on the transfer function of a cylindrical differential electrical mobility classifier (DEMC), a key component in differential mobility sizers. Applying numerical modeling, we found that the inner rod tilt caused variations in the flow and electrical fields in the DEMC classification channel, consequently deteriorating the DEMC transfer function (i.e., reducing the peak and widening the width). In hightilt cases, the single peak of the transfer function was split into two. Further investigation revealed that the alternation of the electrical field in the classification channel (due to rod tilting) was primarily responsible for this deterioration, which was particularly pronounced for a high sheath-to-aerosol flow ratio. However, increasing the total DEMC flow reduced the adverse effects of rod tilting on the transfer function. Finally, increasing both the radii ratio, $\mathrm{R}_{2} / \mathrm{R}_{1}$ (the outer cylinder radius to the inner rod radius), and the classification channel length, L, negatively impacted the transfer function.
\end{abstract}

Keywords: Differential electrical mobility classifier (DEMC); Cylindrical DMA design; Tilted sizing channel.

\section{INTRODUCTION}

The sizing/classification of sub-micrometer-sized particles is primarily accomplished in the aerosol research community by using a differential electrical mobility classifier (DEMC), one of the key components in an electrical mobility sizer. A DEMC differentiates particles based on their electrical mobility. The cylindrical type is predominant among various DEMC designs because of no sidewall presence in the particle classification channel (Müschenborn, 2007). A cylindrical DEMC is basically composed of an inner rod and an outer cylinder coaxially aligned. The annular spacing between the inner rod and outer cylinder defines the particle classification channel, in which the clean sheath gas flows and serves as the barrier for charged particles introduced from the outer cylinder. Having an electrical field set up in the annular flow channel, charged particles cross the sheath flow barrier and move towards the inner rod. Downstream of the classification channel, a circular exit slit is designed in the inner rod to extract classified particles out from the classification channel. Relative to the exit slit, particles with high electrical mobility are deposited onto the upper portion of the rod, and ones with low mobility are

\footnotetext{
* Corresponding author.

E-mail address: dechen3@vcu.edu
}

either deposited onto the lower part of the rod or carried out of the channel by excess flow. Only particles within a narrow mobility range are extracted from the exit slit.

All the DEMCs were designed and constructed with the assumption that the DEMC would be perfectly assembled. In reality, the imprecise machining of parts could make it imperfect in the DEMC assembly. Two types of basic geometrical imperfection have been observed in the DEMCs (if not carefully designed/assembled): axial misalignment and tilt of the inner rod relative to the outer cylinder. To the authors' knowledge, limited attention has been paid to investigate the effect of axial misalignment and tilt on the performance of a cylindrical DEMC (i.e., transfer function). However, the fundamental knowledge on how significantly the geometrical imperfection affects the DEMC performance is essential to the DEMC design.

The performance of an eccentric DEMC had been analyzed and given in the appendix of Knutson's dissertation (1972). Intensive investigation on the effect of axial misalignment on the performance of a cylindrical DEMC has been reported by the authors (Alsharifi and Chen, 2019). In the work of Knutson (1972), the flow and electrical fields in the annular classification channel of a DEMC were considered 2-D (i.e., fully developed for the flow). Only non-diffusive particles were considered. Via numerical modeling, Alsharifi and Chen (2019) have investigated the eccentric DEMC performance under various design and operation conditions. However, no study on the effect of axial tilt on 
the performance of a cylindrical DEMC has been reported. Different from that in an eccentric DEMC, the flow and electrical fields in the classification channel of a cylindrical DEMC are completely 3-D under the axial tilt condition. It is because the cross-section of the particle classification channel remains varied along the axial direction of a DEMC, resulting in the continuous flow developing in the tilted DEMC classification channel. Notice that the flow in the classification channel of an eccentric DEMC will eventually become fully developed so long as the channel length is sufficient. Thus, the effect of inner rod tilt on the DEMC performance is expected to be different from that of axial misalignment.

The objective of this study is to investigate the effect of inner rod tilt on the performance of a cylindrical DEMC. The numerical modeling approach was applied in this study. Our modeling of the DEMC performance was set up via the combination of COMSOL Multiphysics $5.4^{\circledR}$ and MATLAB R2018 $\mathrm{a}^{\circledR}$. Prior to the parametric study, the setup model was verified by the comparison of numerical results with theoretical ones (Knutson and Whitby, 1975; Stolzenburg and McMurry, 2008), and the experimental work of Chen et al. (1998). The parametric study was then performed to investigate the effect of geometrical variables on the transfer function of cylindrical DEMCs. The studied geometrical and flow variables included the tilting angle, the classification channel length, the outer to inner radius ratio, cross-sectional area, and the aspect ratio (i.e., channel length: hydraulic diameter of the annular spacing). The effect of sheath-to-aerosol flow ratio, $\beta$, on the performance of tilted DEMCs was also investigated.

\section{NUMERICAL MODELING OF CYLINDRICAL DEMCS}

Both COMSOL Multiphysics $5.4^{\circledR}$ and MATLAB R2018a ${ }^{\circledR}$ Multiphysics were applied to set up the DEMC model. A typical computational domain to model the performance of a DEMC having the tilted inner rod is shown in Fig. 1 ((a) is the side view, and both (b) and (c) are the selected cross-sectional views). In this study, we define the DEMC inner rod tilting, $\tau$, as the ratio of the angle, $\theta$, between the axis of the inner rod and the outer cylinder to the maximal tilting angle, $\theta_{\mathrm{m}}$, which depends on the inner rod radius, $\mathrm{R}_{1}$, outer cylinder radius, $\mathrm{R}_{2}$, and the classifying channel length, $\mathrm{L}$. The formula for the $\theta_{\mathrm{m}}$ calculation is given in the supplementary information (SI). Polydisperse particles flow into the particle classification channel from the particle entrance slit at the outer cylinder, and classified particles leave the channel from the exit slit at the inner rod. A clean sheath flow enters the classification channel from the top and the excess flow exits the channel from the bottom exit. A high voltage was applied on the inner rod and the outer was electrically ground.

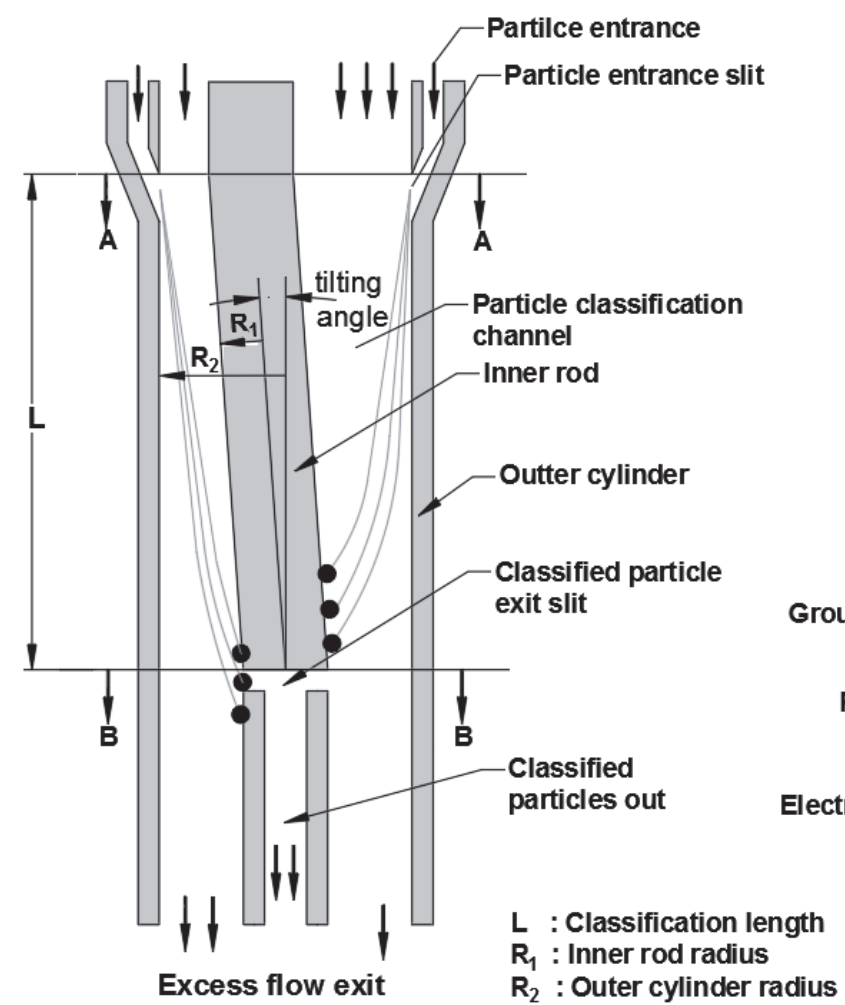

(a)

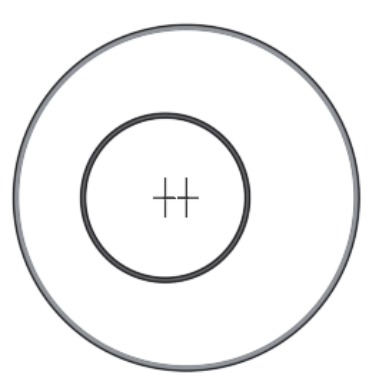

(b) A-A

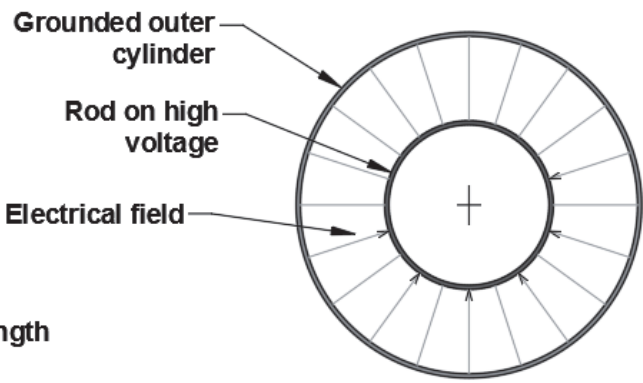

(c) B-B

Fig. 1. The schematic diagram of a cylindrical differential electrical mobility classifier (DEMC) with a tilted inner rod and the computational domain used in the DEMC modeling: (a) the side view (with the illustration of the particle movement in the classification channel); (b) the cross-sectional view at the location near the particle entrance; and (c) the cross-sectional view at the location near the particle exit silt. 
Steady and incompressible Navier-Stokes and continuity equations were applied to calculate the flow field in the classification channel of a DEMC. The Laplace equation was used to calculate the electrical potential, $\varnothing$, in the DEMC's classification channel. The intensity of the electrical field, $\vec{E}$, was derived by $\vec{E}=-\nabla \varnothing$.

For the flow field calculation, the velocity profile at the sheath flow entrance was assumed to be uniform because the channel flow would not be fully developed in the studied channel (due to the continuous variation of channel cross-section). A uniform velocity profile was assumed at the classified particle exit (because of the installation of the flow straightener). The pressure boundary condition was set at the excess flow exit. No-slip boundary conditions were applied to all the solid walls in contact with fluid flow. For the electrical field, the inner rod was set at an elevated voltage, and the outer cylinder was on the electrical ground.

The performance of a DEMC is characterized by its transfer function, defined as the probability of a particle with a given electrical mobility $\left(Z_{p}\right)$ entering the DEMC classification region and reaching the classified aerosol exit. The calculation of the transfer function of a DEMC for singly charged particles of a given size is based on the particle flux. The detail can be found in the work of Alsharifi and Chen (2019). The method is briefly summarized herein: With the assumption of uniform particle concentration at the particle entrance, the transfer function of a DEMC could be calculated as:

$$
\Omega=\frac{\sum_{1}^{n} p_{i} w_{i} A_{i}}{\sum_{1}^{n} w_{i} A_{i}}
$$

where $\Omega$ is the transfer function of a DEMC for particles of a given size (carrying single electrical charge); $n$ is the total number of meshes at the aerosol entrance for releasing particles; $w_{i}$ is the flow velocity at the released particle location; $A_{i}$ is the area of the $i^{\text {th }}$ mesh, and $p_{i}$ is the probability of particles released from the centroid of the mesh, $i$, and penetrating through the particle classification channel of a DEMC. For non-diffusive particles, the value of $p_{i}$ is 1.0 if a presentative particle is released from the centroid of the mesh, $i$, and penetrates through the classification channel and is 0 if the particle is either deposited or carried out by excess flow.

Table 1 summarizes the values of the physical properties of gas and particles used in our modeling. The effect of axial tilting on the performance of a cylindrical DEMC was investigated for particles with the sizes of 160 , 340 , and $815 \mathrm{~nm}$ and under the condition of different sheath-to-aerosol flow ratios, $\beta$, of $4,5,10$, and 20 (i.e., both sheath and particle flow rates were varied in 5.0 $20 \mathrm{~L} \mathrm{~min}^{-1}$ and in $0.5-4 \mathrm{~L} \mathrm{~min}^{-1}$, respectively). Table 2 summarizes the ranges of DEMC dimensions studied. Note that the DEMC dimensions under the study were selected using the dimensions of both electrical classifiers, offered by TSI Inc.

\section{RESULT AND DISCUSSION}

\section{Distorted Flow and Electrical Fields in a Tilted DEMC Classification Channel}

Fig. 2 shows a distorted flow field in the particle classification channel of a cylindrical DEMC having the tilted inner rod. For better visualization, the DEMC with 50\% inner rod tilt was selected. The selected DEMC, operated at the particle and sheath flow rates of 1 and $10 \mathrm{~L} \mathrm{~min}^{-1}$, has the inner rod and outer cylinder radii of 1 and $2 \mathrm{~cm}$, respectively and the channel length of $12 \mathrm{~cm}$. Because of the tilting of the inner rod, the channel cross section is varied along the primary flow direction. The side view of

Table 2. The geometrical dimensions/parameters of DEMCs studied in this work.

\begin{tabular}{ll}
\hline Geomtrical Parameter & Range \\
\hline Inner Rod Radius, $\mathrm{R}_{1}$ & $0.5-2[\mathrm{~cm}]$ \\
Outer Cylinder Radius, $\mathrm{R}_{2}$ & $1.2-4[\mathrm{~cm}]$ \\
Classification Channel Length, $\mathrm{L}$ & $5-45[\mathrm{~cm}]$ \\
Radial Ratio $\left(\mathrm{R}_{2} / \mathrm{R}_{1}\right)$ & $2-4$ \\
Aspect Ratio $\left(\mathrm{L} / \mathrm{R}_{1}\right)$ & $2.5-10$ \\
\hline
\end{tabular}

Table 1. The physical properties of gas and particles used in this DEMC performance modeling.

\begin{tabular}{|c|c|c|}
\hline & Constant & Values and Formulas \\
\hline \multirow[t]{5}{*}{ Particle } & Diameter, $D_{p}$ & $160,340,815 \mathrm{~nm}$ \\
\hline & Mass density, $\rho_{p}$ & $1000 \mathrm{~kg} \mathrm{~m}^{-3}$ \\
\hline & Slip correction factor, $C_{c}$ & $\left(\quad-\frac{0.596}{1}\right)$ \\
\hline & & $C_{c}=1+K n \mid 1.155+0.471 e^{K n}$ \\
\hline & Elementary charge, e & $1.602 \times 10^{-19} \mathrm{C}$ \\
\hline \multirow[t]{5}{*}{ Air } & temperature, $\mathrm{t}$ & $293.15 \mathrm{~K}$ \\
\hline & dynamic viscosity, $\mu$ & $18.5 \times 10^{-6} \mathrm{~N} . \mathrm{s} \mathrm{m}^{-2}$ \\
\hline & density, $\rho$ & $1.2047 \mathrm{~kg} \mathrm{~m}^{-3}$ \\
\hline & mean free path, $\lambda$ & $0.066[\mu \mathrm{m}]$ \\
\hline & Boltzmann constant & $1.38064852 \mathrm{e}-23[\mathrm{~J} / \mathrm{K}]$ \\
\hline \multirow[t]{2}{*}{ Flow } & Aerosols inlet and exit flow & $0.5-4.0 \mathrm{~L} \mathrm{~min}^{-1}$ \\
\hline & Sheath flow rate range & $5.0-20.0 \mathrm{~L} \mathrm{~min}^{-1}$ \\
\hline
\end{tabular}




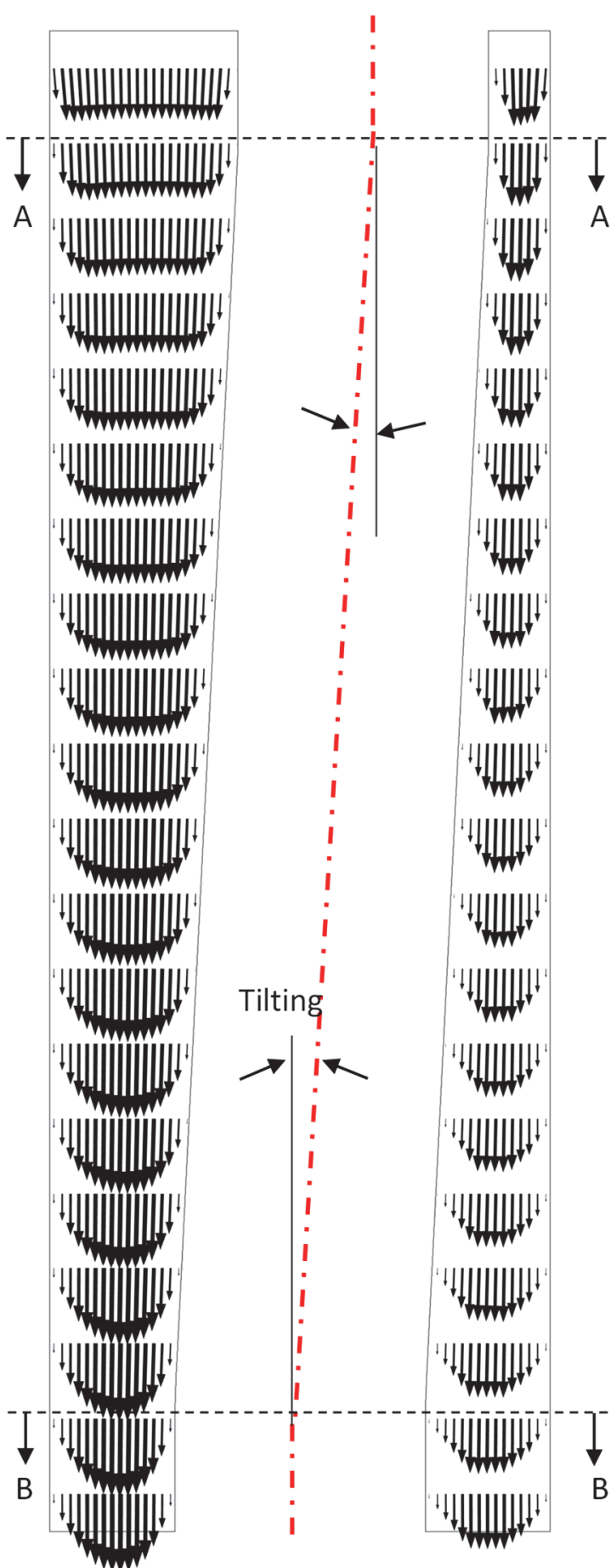

(a)

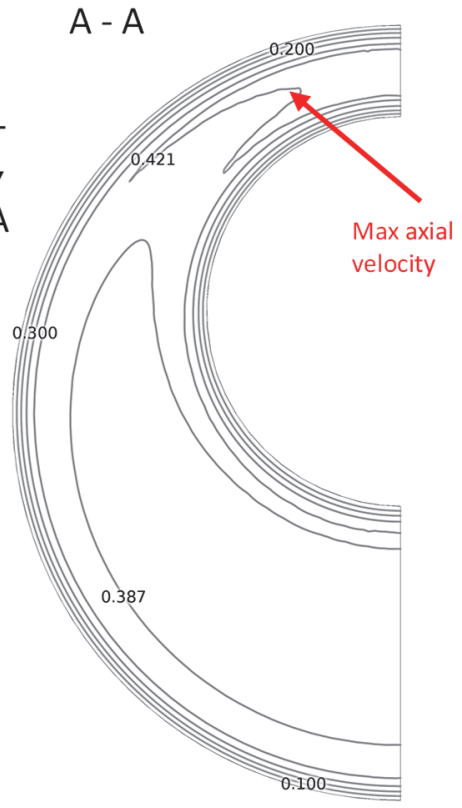

B - B

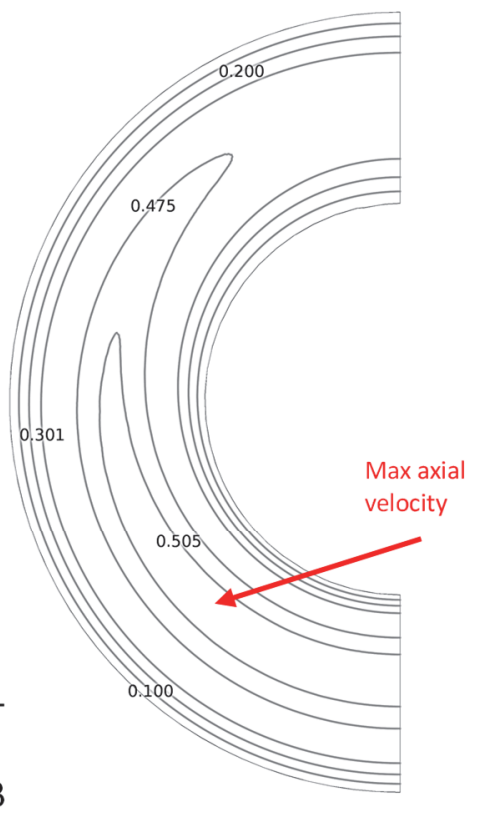

(b)
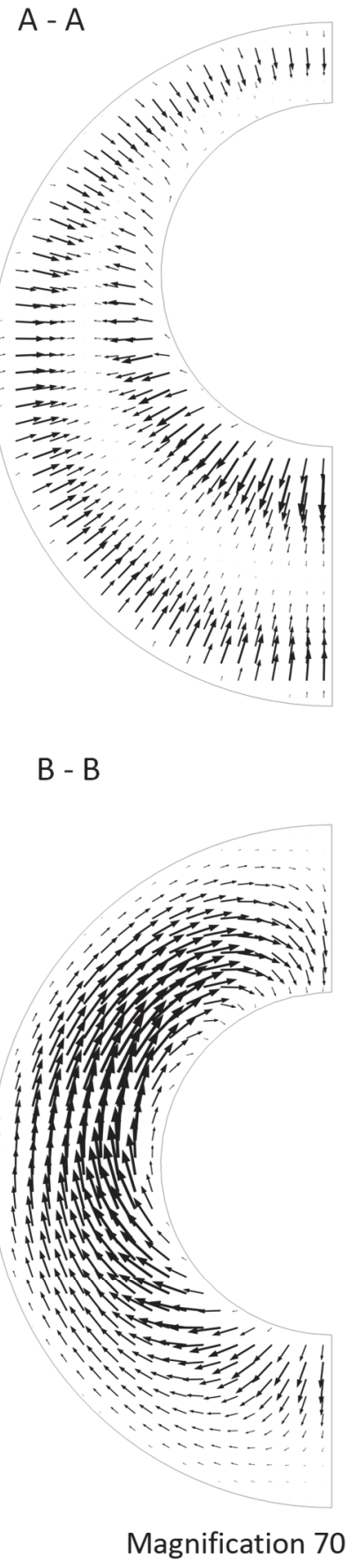

(c)

Fig. 2. The illustration of flow field in the classification channel of a cylindrical DEMC having outer and inner cylinder radii of 1 and $2 \mathrm{~cm}$, respectively, and a length of $12 \mathrm{~cm}$. The relative tilting of the inner rod is $50 \%$. The DEMC is operated at the sheath flow rate of $10 \mathrm{~L} \mathrm{~min}{ }^{-1}$. The selected cross sections, A-A, and B-B, are located at 1 and $11 \mathrm{~cm}$, measured from the sheath flow entrance, respectively: (a) the side view showing the axial velocity profiles along the particle classification channel; (b) the contour of the axial velocity component; and (c) the circumferential velocity component (with the magnification of 70).

the DEMC classification channel illustrates the convergent (left) and divergent (right) spacing on both sides of inner rod. Fig. 2(a) gives the axial flow velocity profiles along the convergent and divergent spacings. As expected, in each spacing, the velocity component in the axial direction developed into a parabolic-like profile (i.e., having the maximal velocity and 0 at the wall) but the profile varies in the axial direction. Fig. 2(b) plots the contours of axial 
velocity component at two selected cross sections (i.e., A-A near the flow entrance and B-B near the flow exit). Because of uneven spacing on both sides of the inner rod, the location of maximal axial velocity shifted from the narrowest gap to the widest gap. It is because the pressure drop is higher for the flow moving in the narrow gap as compared to that in wide gap.

Fig. 2(c) shows the radial and circumferential velocity vector fields at two selected cross sections, A-A and B-B (at the same levels as those in Fig. 2(b)). Note that, for the illustration, the magnitude of shown velocities was magnified 70 times. At the A-A section the velocity is primarily in the radial direction because of flow developing from the uniform profile to the parabolic-like one. At the B-B section, the circumferential velocity component became higher compared to that at the A-A section. It is because of the flow moving toward the wider gap (due to lower pressure drop for axial flow). As the axial flow increased in the wide gap and decreased in the narrow gap, the secondary flow at each cross section of the DEMC classification channel was then developed.

Fig. 3 illustrates the distorted electrical field at the cross section located at the middle level of DEMC classification channel. Because of uneven gap in the circumferential direction of classification channel (due to the inner rod tilting), the electrical potential was intensified in the narrow gap compared to that in the wide spacing.

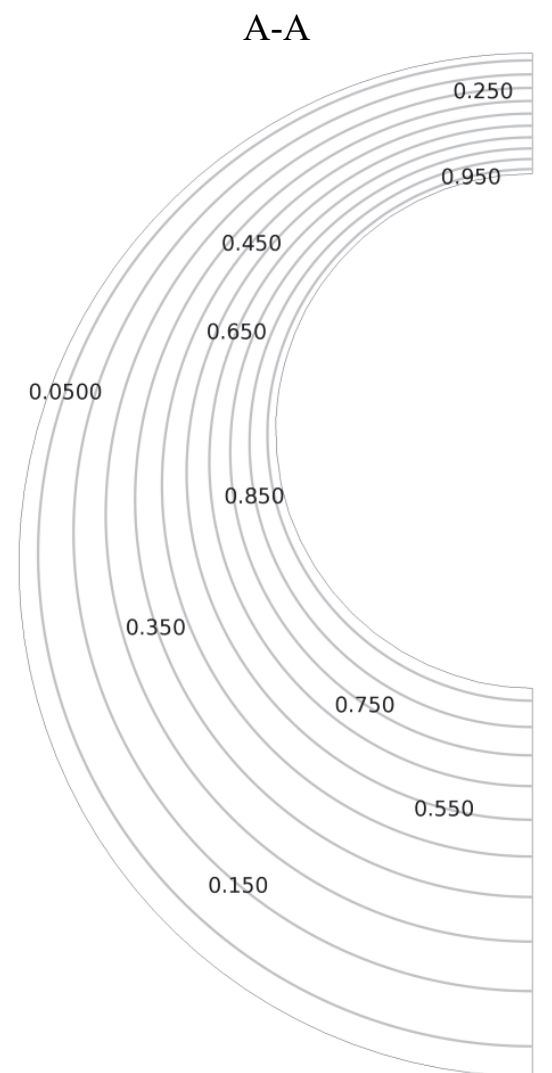

\section{Transfer Function of a DEMC Having a Tilted Inner} Rod

Fig. 4(a) shows the transfer function of a cylindrical DEMC having a tilted inner rod at various tilting percentages $(0 \%, 2 \%, 5 \%, 6 \%, 7 \%$, and $10 \%)$, defined as the ratio of actual tilting angle to the maximal angle. The particle size selected in this part of modeling is $815 \mathrm{~nm}$. The DEMC, operated at the particle and sheath flow rates of 1 and $10 \mathrm{~L} \mathrm{~min}^{-1}$, has the inner rod and outer cylinder radii of 1 and $2 \mathrm{~cm}$, respectively, and the channel length of $25 \mathrm{~cm}$. The shape of the transfer function for the $0 \%$ tilting (i.e., non-tilted DEMC) is triangular (once the electrical mobility, $Z_{p}$, was normalized by the central mobility, $Z_{p}^{*}$ ). With the increase of inner rod tilting, the peak of the DEMC transfer function was decreased, flattened, and eventually double-peaked. In conjunction with the peak change, the width at half of peak height (WHPH) was increased as the inner rod tilting was increased. The deterioration of the transfer function could be explained by understanding the particle fate near the exit slit of the classification channel of studied DEMC.

Fig. 4(b) shows the particle fate near the exit slit of a DEMC with a tilted inner rod after particles were released from the particle entrance slit. The abscissa of Fig. 4(b) is from 0 (for the narrowest gap in the DEMC classification channel) to $\pi$ (for the widest gaps), and reaching to $2 \pi$, which periodically returns to the same coordinate point of 0 .

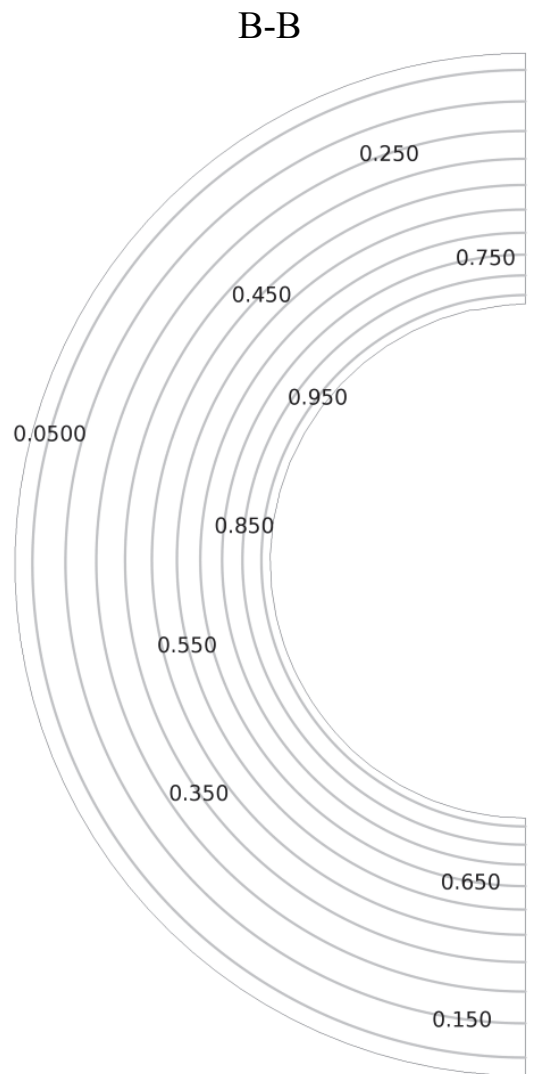

Fig. 3. The illustration of the electrical potential contour (normalized by the voltage applied on the inner rod) located at the middle location of the classification channel of a cylindrical DEMC having outer and inner cylinder radii of 10 and $20 \mathrm{~mm}$, respectively, a classification length of $12 \mathrm{~cm}$, and an inner rod tilt of $50 \%$. 


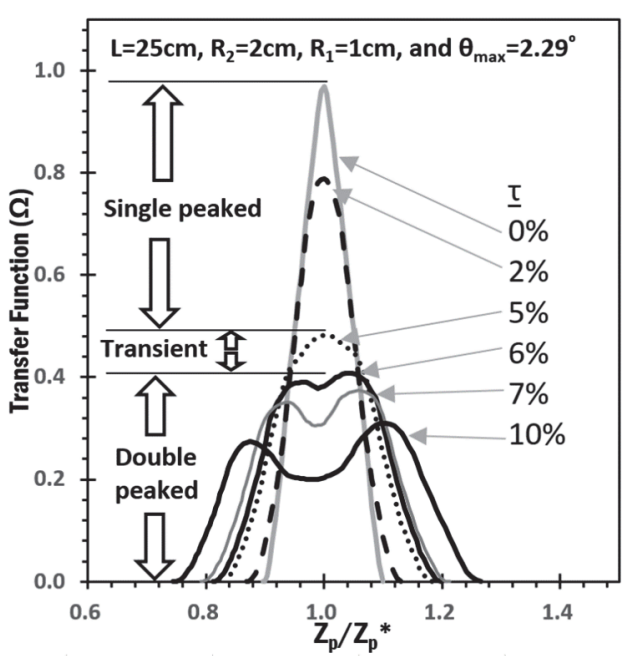

(a)

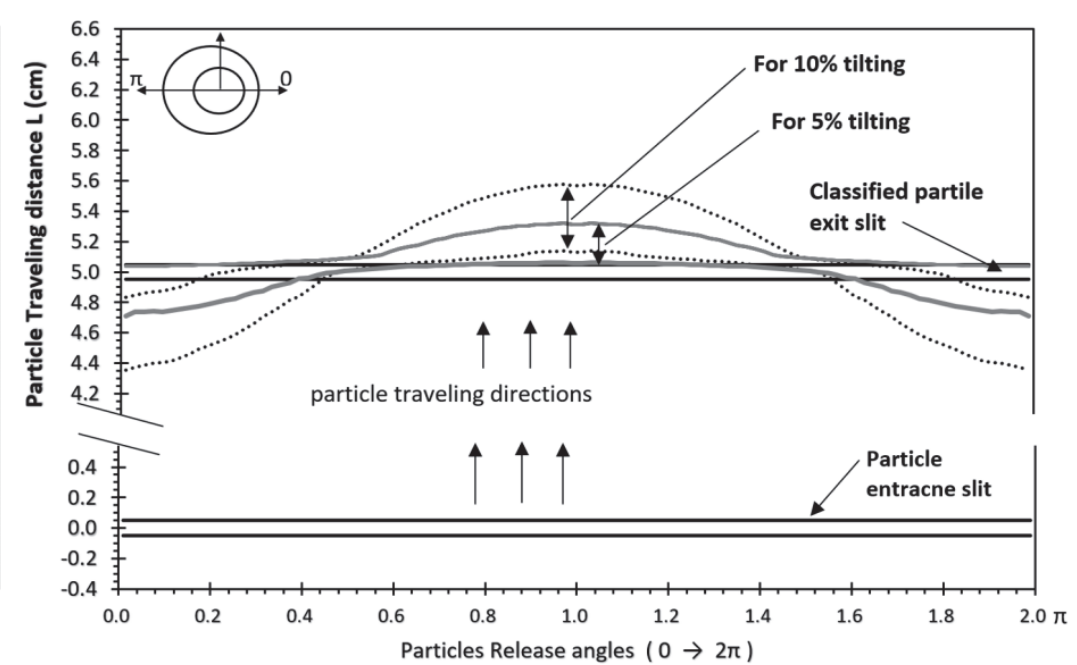

(b)

Fig. 4. (a) The variation in the non-diffusive transfer function of tilted DEMCs having the inner and outer radii of 10 and $20 \mathrm{~mm}$, respectively, and a classification length of $5 \mathrm{~cm}$ as the function of inner rod tilting. The aerosol and sheath flow rates of DEMCs were 1 and $10 \mathrm{~L} \mathrm{~min}^{-1}$, respectively (b) The position of particles in the neighborhood of the classified particle exit slit after they were released from the particle entrance slit of the DEMCs with 5\% and $10 \%$ inner rod tilting. The DEMCs have a classification length of $5 \mathrm{~cm}$, and inner rod and outer cylinder radii of 10 and $20 \mathrm{~mm}$, respectively (operated at the aerosol and sheath flow rates of 1 and $10 \mathrm{~L} \mathrm{~min}^{-1}$, respectively).

Because of the variation of particle travelling distance after entering the classification channel, the particle position when moving close to the classified particle exit slit was varied at different angles. Only particles reaching the region nearby the particle exit slit (between two solid lines plotted in the figure) are expected to exit the classification channel. As shown in Fig. 4(b), the location variation of particles in the neighborhood of the exit slit was increased with the increase of inner rod tilting, resulting in the increase of particle loss in the classification channel. The variation status of particle position nearby the classified particle exit slit also depended on the electrical mobility of particles: less variation for particles with high electrical mobility compared to that for ones with low electrical mobility. It is why the peak in the low mobility range is lower than that in the high mobility range once the peak of the transfer function is split.

Both the flow and electrical field distortion in the particle classification channel of a DEMC with tilted inner rod contributed to the deterioration of the transfer function. In this part of study, we would like to identify the dominant factor influencing the shape of the DEMC transfer function. Fig. 5 shows the transfer functions calculated with the assumptions of uniform velocity profiles at the sheath flow entrance only and in the entire classification channel for a cylindrical DEMC with the relative tilting of $5 \%$ and $10 \%$, operated at the aerosol and sheath flow rates of 1 and $10 \mathrm{~L} \mathrm{~min}^{-1}$, respectively. The particle classification length of studied DEMCs was $5 \mathrm{~cm}$ and the particle size was $160 \mathrm{~nm}$. The difference between the two calculated transfer functions could be attributed to the effect of flow field distortion. By comparison, it is found that the shape and peak split of the transfer function for a DEMC with a tilted inner rod is primarily due to the distortion of the electrical field. The distortion of the flow field makes the width of the transfer function broader.

\section{Tilted DEMC Transfer Function at Different Sheath-to- aerosol Flow Ratios and Total Flow Rates}

The effect of sheath-to-aerosol flow ratio $(\beta)$ on the transfer function of a DEMC having a tilted inner rod was investigated. The studied DEMC with the $10 \%$ tilting has the classification length of $25 \mathrm{~cm}$, and the inner and outer cylinder radii of 10 and $20 \mathrm{~mm}$, respectively. Fig. 6(a) shows the numerical transfer functions of studied DEMCs, operated at $\beta=5,10$, and 20 . It is noticed that the effect of inner rod tilting on the transfer function of a DEMC is more pronounced when operated at high sheath-to-aerosol flow ratio (i.e., at high sizing resolution power) as compared with that at low $\beta$.

Fig. 6(b) shows the numerical transfer function of a cylindrical DEMC with the $10 \%$ inner rod tilting and the particle classification length of $25 \mathrm{~cm}$ (operated at the sheath-to-aerosol flow ratio of 5 under different total flow rates). It is found that the reduction of total flow rate, while keeping the sheath-to-aerosol flow ratio constant, increased in the particle loss, resulting in the reduction of transfer function. It is because of flow velocity reduction at the classified particle exit slit (to overcome the electric force for exiting).

\section{Effect of the Geometrical Parameters of Classification Channel on Tilted DEMC Transfer Function}

The classification channel of a cylindrical DEMC can be characterized by the inner rod and outer cylinder radii, $\mathrm{R}_{1}$ and $\mathrm{R}_{2}$, respectively, and the length, $\mathrm{L}$. It is beneficial to generalize our results by studying the effect of two dimensionless factors, i.e., the ratio of outer cylinder to the 


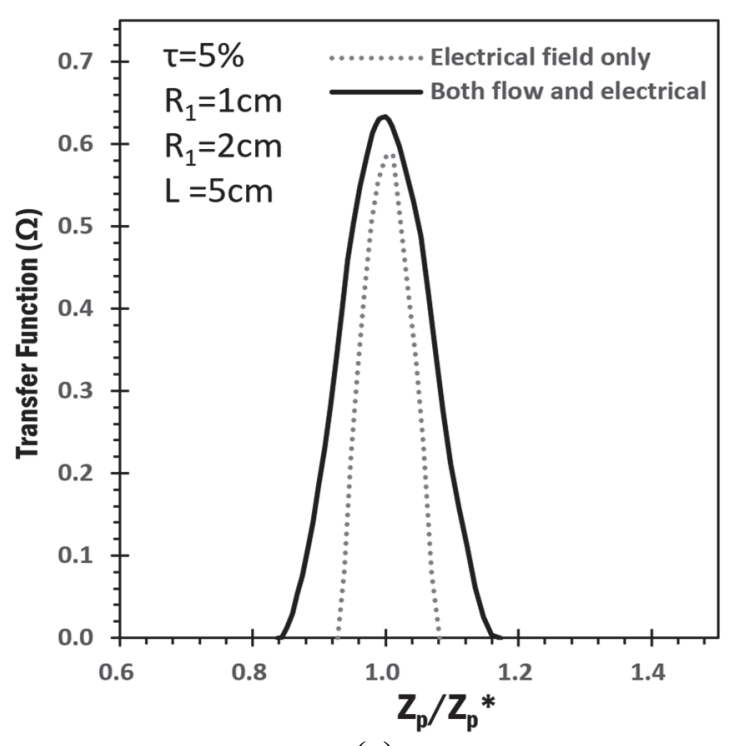

(a)

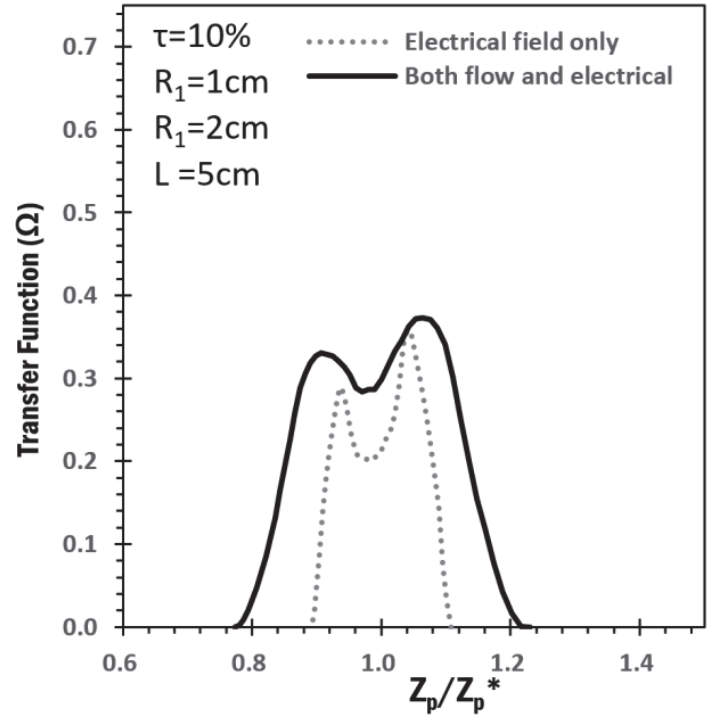

(b)

Fig. 5. Comparison of the numerical transfer function of a DEMC having inner and outer cylinder radii of 10 and $20 \mathrm{~mm}$, respectively, and a classification length of $5 \mathrm{~cm}$. The particle size was $160 \mathrm{~nm}$. The aerosol and sheath flow rates of the studied DEMCs were 1 and $10 \mathrm{~L} \mathrm{~min}^{-1}$, respectively, for (a) 5\% inner rod tilting and (b) $10 \%$ tilting.

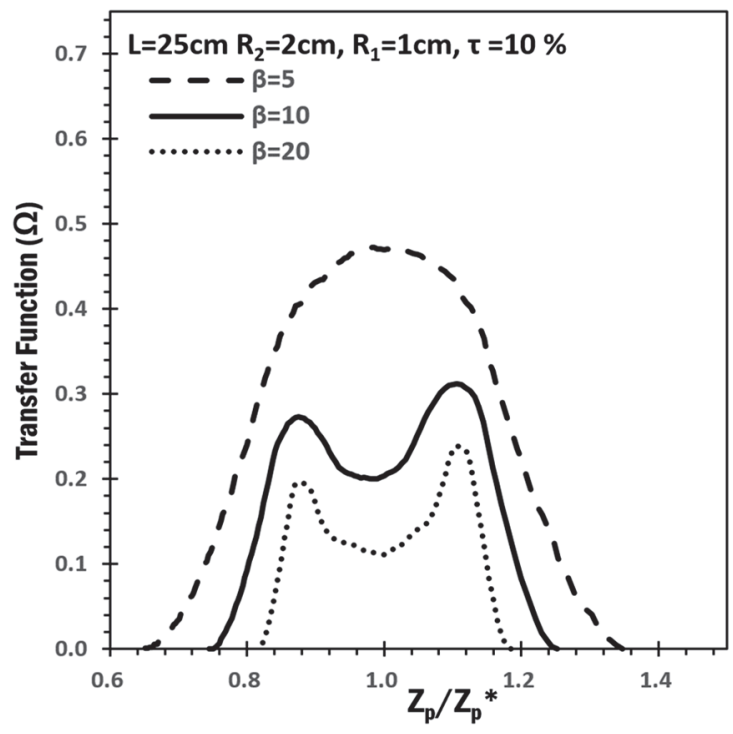

(a)

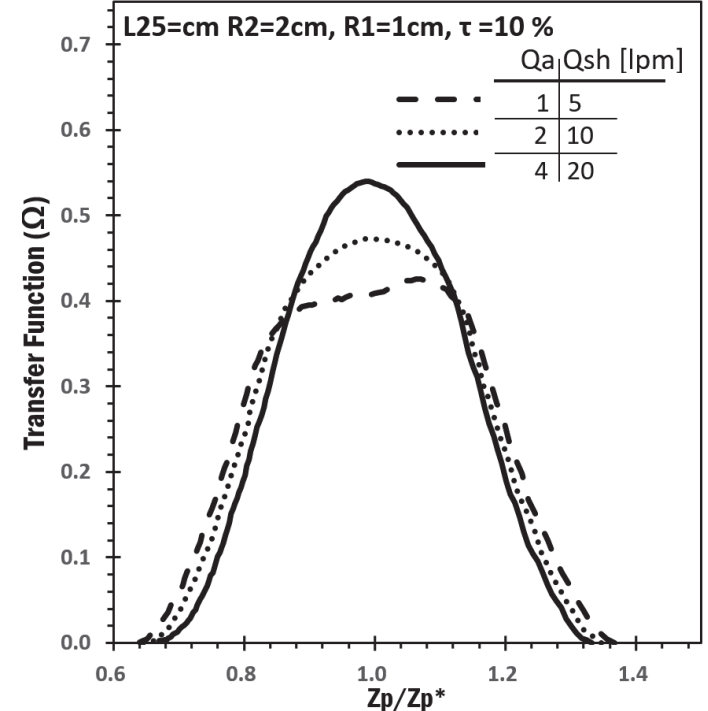

(b)

Fig. 6. (a) The variation in the transfer function of a DEMC with $10 \%$ inner rod tilting at the sheath-to-aerosol flow ratios, $\beta$, of 5,10 , and 20 (for a sheath flowrate of $10 \mathrm{~L} \mathrm{~min}^{-1}$ and particle flowrate of 2 , 1 , and 0.5 , respectively). (b) The variation in the transfer function of the above DEMC operated at different total flowrates while keeping the $\beta$ value at 5 .

inner rod radii, $\mathrm{R}_{2} / \mathrm{R}_{1}$, and the aspect ratio of the inner rod, $\mathrm{L} / \mathrm{R}_{1}$, on the tilted DEMC transfer function. Note that the selection of above two parameters was because they define the maximal tilting angle in a DEMC (given in SI).

Fig. 7(a) gives the numerical transfer functions for the DEMCs with $10 \%$ inner rod tilting, the particle classification length of $5 \mathrm{~cm}$, and radii ratios of 2,3 , and 4 (for the $R_{2}: R_{1}$ ratios of $2: 1,3: 1$, and $4: 1 \mathrm{~cm}$, respectively). The particle and sheath flow rates applied in the studied DEMC were 1 and $10 \mathrm{~L} \mathrm{~min}^{-1}$. It is evidenced that, for the same tilting, the increase of the radii ratio (leading to the increase of the cross-sectional area of classification channel and actual tilting angle) decreased the height of tilted DEMC transfer function. Fig. 7(b) presents the calculated transfer functions of DEMCs with the $10 \%$ tilting, particle classification length of $5 \mathrm{~cm}$, and radii ratio of 2 while varying the rod aspect ratios (i.e., 2.5, 5, 7.5, and 10). It is found that the effect of the rod aspect ratio on the tilted DEMC transfer function was negligible at the rod aspect ratio higher than 5 for given flow rates. However, at the low rod aspect ratio of 2.5 , the height of the transfer function is significantly lower than those at higher aspect 


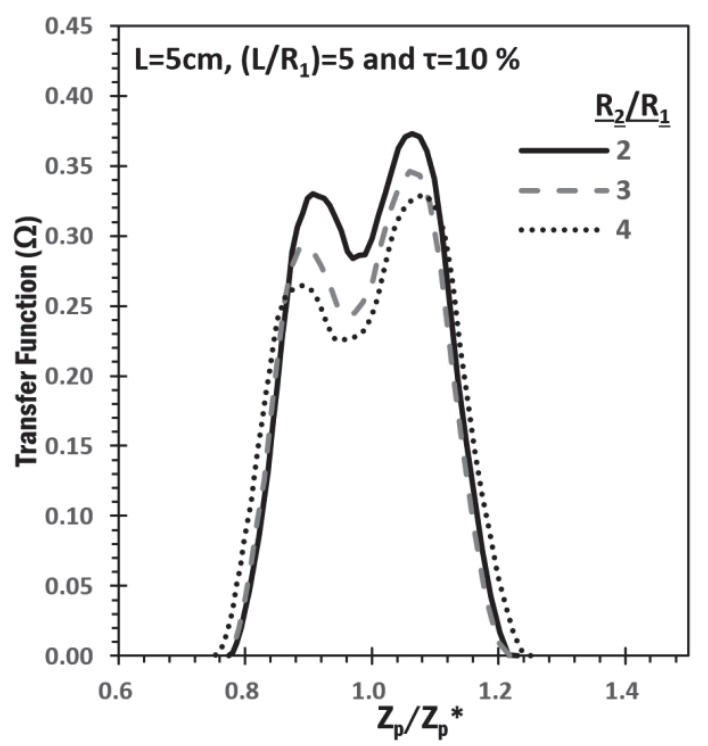

(a)

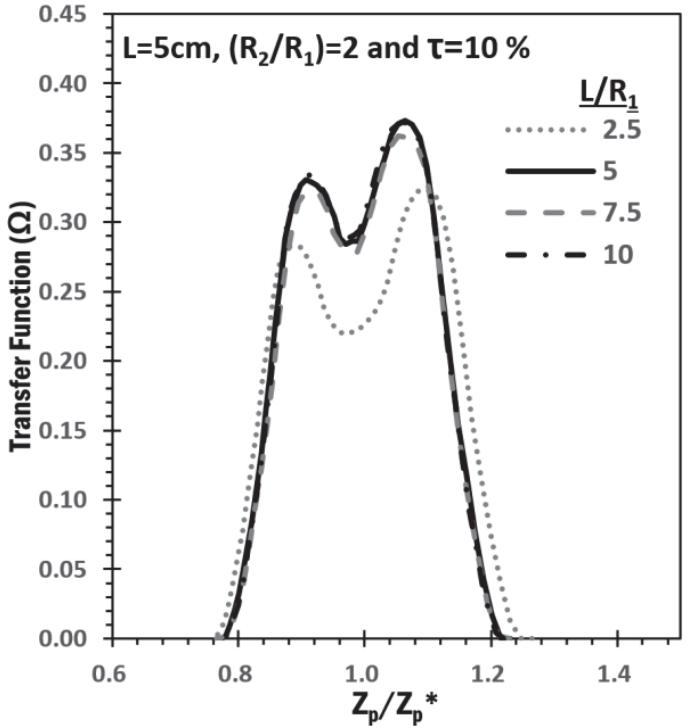

(b)

Fig. 7. The effect of DEMC geometrical parameters on the transfer function of DEMCs with $10 \%$ tilted inner rods while keeping the particle classification length of $5 \mathrm{~cm}$ by (a) varying the radii ratios, $R_{2} / R_{1}$ of 2,3 and 4 , while keeping the rod aspect ratio, $L / R_{1}$ of 5 , and (b) changing the rod aspect ratio, $L / R_{1}$ of $2.5,5,7.5$, and 10 , while keeping the radii ratio of 2 . DEMCs were operated at the particle and sheath flowrates of 1 and $10 \mathrm{~L} \mathrm{~min}^{-1}$.

ratios. It is because, at the low rod aspect ratio, the actual tilting angle for inner rod was larger than that at high rod aspect ratio for the same tilting defined in this work.

\section{Classification of the Tilted DEMC Transfer Function}

For a given DEMC operated at the fixed rates of particle and sheath flows, the shape of the transfer function is varied as the inner rod tilt is increased (as shown in Fig. 4(a)). The shape variation of the DEMC transfer function depends on both the sheath-to-aerosol flow ratio and the length of the classification channel. Accordingly, the evolution of transfer function shape for a cylindrical DEMC due to the inner rod tilting could be classified into three regions: single-peaked, transient, and double-peaked. Fig. 8(a) shows the change of the single-peaked, transient, and double-peaked transfer function regions as the function of classification channel length for a DEMC operated at the $\beta$ value of 10 . Fig. $8(\mathrm{~b})$ shows the change of the aforementioned transfer function regions as the function of the sheath-to-aerosol flow ratio for a DEMC with the classification length of $25 \mathrm{~cm}$. Note that the studied DEMC has the inner rod and outer cylinder radii of 10 and $20 \mathrm{~mm}$.

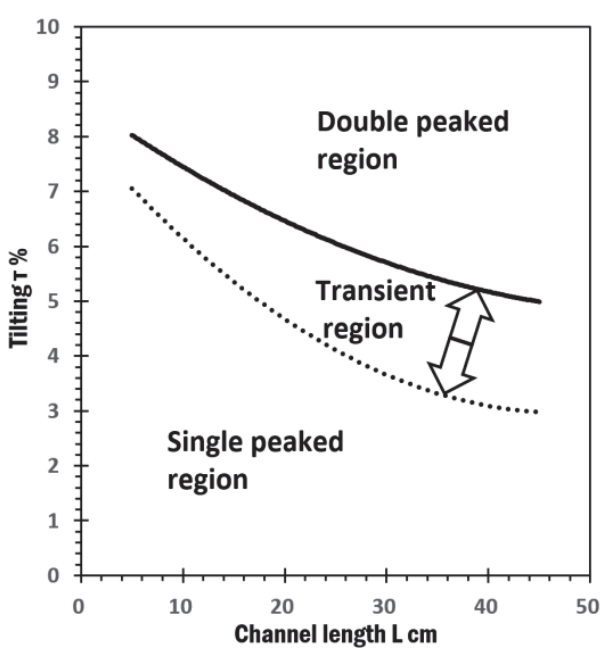

(a

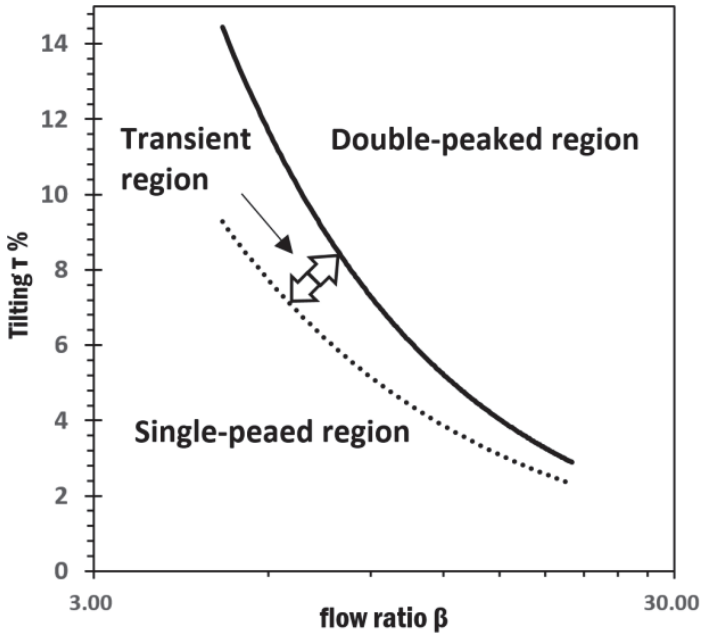

(b

Fig. 8. Regions for single-peaked, transient, and double-peaked transfer functions of DEMCs with tilted inner rods mapped on the relative tilting (a) vs. classification length, L, domain for DEMCs, operated at the $\beta$ value of 10 and (b) vs. sheath-to-aerosol flow ratio, $\beta$, domain for DEMCs, with a classification length of $25 \mathrm{~cm}$. All the studied DEMCs had inner rod and outer cylinder radii of 10 and $20 \mathrm{~mm}$, respectively. 
It is evidenced in Fig. 8 that the tilting of the inner rod shall be tightly controlled for a cylindrical DEMC having a long classification channel length and operated at high sheath-to-aerosol flow ratio (for high sizing resolution).

\section{CONCLUSION}

The performance of a cylindrical differential electrical mobility classifier (DEMC) with a tilted inner rod was numerically investigated. Our results provide critical insight into the effect of rod tilting on the transfer function of a cylindrical DEMC. A tilted inner rod was found to distort both the flow and the electrical fields, leading to an imbalance in the axial flow velocity component, an increase in the circumferential velocity component, and asymmetry in the field intensity, in the particle classification channel. Consequently, the DEMC transfer function exhibited a reduction in peak height and an increase in the width at half-peak (lower sizing resolution). With extreme tilting, the single peak split into double peaks. As a result, the particles near the exit slit varied in position, depending on where they had been released, along the slit, increasing particle loss in the classification channel. Further investigation identified the distortion in the electrical field as the dominant factor in shaping the transfer function.

Furthermore, increasing the total flow rate and decreasing the classification channel length mitigated the adverse effects of inner rod tilting on the transfer function, which were especially obvious with a high DEMC sheath-toaerosol flow ratio. The ratio of the outer cylinder radius to the inner rod radius also affected performance (when the relative tilt remained constant), but the aspect ratio (of the channel length to the inner rod radius) became a factor only when it fell below 2.5 (due to the increase in the absolute tilting angle of the inner rod).

To describe the change in shape for the DEMC function as the relative tilt of the inner rod varied, we identified three regions, viz., single-peaked, transitional, and doublepeaked. Given a constant classification channel length and sheath-to-aerosol flow ratio, a single peak occurred when the tilt was low, transitioning to a double peak as the tilt increased. However, the transient range broadened when the length of the classification channel increased, whereas it narrowed when the sheath-to-aerosol flow ratio increased.

\section{ACKNOWLEDGEMENT}

The author, T. Alsharifi, appreciates the financial support by HCED Iraq scholarship program.

\section{SUPPLEMENTARY MATERIAL}

Supplementary data associated with this article can be found in the online version at http://www.aaqr.org.

\section{REFERENCES}

Alsharifi, T. and Chen, D.R. (2019). Effect of axial eccentricity on the performance of a cylindrical differential mobility classifier. Aerosol Sci. Technol. 53: 735-748. doi: 10.1080/02786826.2019.1599097.

Chen, D.R., Pui, D.Y.H., Hummes, D., Fissan, H., Quant, F.R. and Sem, G.J. (1998). Design and evaluation of a nanometer aerosol differential mobility analyzer (NanoDMA). J. Aerosol Sci. 29: 497-509. doi: 10.1016/S00218502(97)10018-0

Knutson, E.O. (1972). The distribution of electric charge among the particles of an artificially charged aerosol. Mechanical Engineering, Ph. D. University of Minnesota, Minnesota.

Knutson, E.O. and Whitby, K.T. (1975). Aerosol classification by electric mobility: Apparatus, theory, and applications. J. Aerosol Sci. 6: 443-451. doi: $10.1016 / 00$ 21-8502(75)90060-9.

Müschenborn, P.M. (2007). Development of a differential mobility particle sizer applied to industrial gas phase synthesis processes for nanoscaled powders, 15-41. http://duepublico.uni-duisburg-essen.de/servlets/Derivate Servlet/Derivate-16630/Dissertation_Mueschenborn.pdf

Stolzenburg, M.R., and McMurry, P.H. (2008). Equations governing single and tandem DMA configurations and a new lognormal approximation to the transfer function. Aerosol Sci. Technol. 42: 421-432. doi: 10.1080/027868 20802157823. 